\title{
EDITORIAL
}

\section{The uncertainties of bronchoalveolar lavage}

\author{
R.P. Baughman
}

Heisenberg noted that a more accurate determination of one quantity will result in a less precise measurement of another. He proposed this principle to describe the difficulties encountered in quantum mechanics, but it has been encountered in all branches of science. For example, placing a stethoscope on a patient's chest wall to hear better will lead to changes in the patient's respiratory pattern. This leads to different sounds heard on auscultation. In performing invasive procedures, the effect can be more dramatic and, unfortunately, is not predictable.

Bronchoalveolar lavage (BAL) has been performed as a research and clinical procedure for more than $20 \mathrm{yrs}$ [1]. It has proved a useful method to sample lower respiratory secretions. This sampling technique has increased our diagnostic sensitivity for infectious and noninfectious lung diseases. In inflammatory lung diseases, it has provided a method of measuring protein and cellular influx into the lung. The cascades of cytokines that occur in the lung during adult respiratory distress syndrome (ARDS) or sarcoidosis have been readily demonstrated by BAL.

One difficulty of lavage is the variability in the recovery of fluid during the procedure. Figure 1 is a schematic representation of the lavage process. The fluid is instilled into the distal airway, which is the equivalent of a sac. This introduced fluid mixes with the air and lung lining fluid. Contamination of the alveolar space by upper airway secretion is possible, but is, apparently, a small problem. This may be due to the small volume of potential contamination from the upper airways (1-2 mL) versus the large volume of fluid instilled dur-

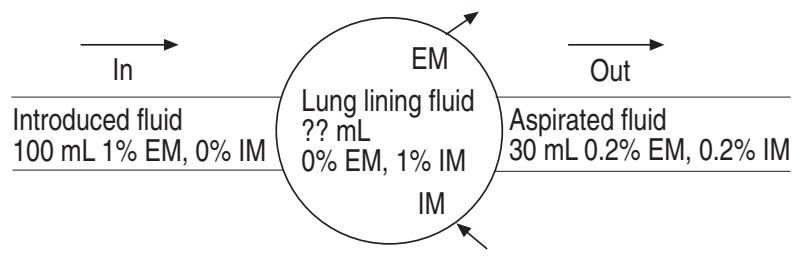

Fig. 1. - Schematic representation of bronchoalveolar lavage (BAL). Markers to determine dilution of BAL include internal and external markers. Their use can be illustrated by an external marker (EM) with a concentration of $1 \%$ in the introduced fluid (IF), $0 \%$ in lung lining fluid (LLF), and $0.2 \%$ in the aspirated fluid (AF). In $30 \mathrm{~mL}$ of $\mathrm{AF}$, there will be $6 \mathrm{~mL}$ of LLF. An internal marker (IM) which has a concentration of $1 \%$ in LLF can be used to measure LLF. Assuming a concentration of IM of $0 \%$ in IF and $0.2 \%$ in AF, there is $6 \mathrm{~mL}$ of LLF in $30 \mathrm{~mL}$ of AF. These calculations assume that no EM leaves or IM enters the alveolar space during the lavage process.

Correspondence: R.P. Baughman, Pulmonary and Critical Care Medicine, University of Cincinnati Medical Center, PO Box 670564, Cincinnati, $\mathrm{OH}, \mathrm{USA}$ ing the lavage (100-200 mL). The amount of mixing in the alveolar space has been difficult to determine, especially since the volume retrieved is never equal to the volume instilled. This incomplete retrieval of fluid means that the aspirated fluid contains an unknown amount of lung lining fluid.

An external marker in the instilled fluid is one method of determining the volume of lung lining fluid that has been aspirated. Dilution of this external marker has been used in a similar manner to the helium dilution technique used to calculate lung volumes. By measuring the concentration of the marker in the instilled and aspirated fluid as well as the volume of aspirated fluid, it is possible to calculate the volume of lung lining fluid aspirated. A major assumption regarding the external marker is that it is not absorbed by the lung surface membrane. Helium has proved to be useful for measuring lung volumes since there is minimal absorption of this inert gas. Methylene blue was proposed as an external marker for measuring lung lining fluid volume [2]. However, methylene blue is taken up by macrophages and epithelial cells. Therefore, the concentration of methylene blue is reduced by a nondilutional pathway and the amount of lung lining fluid will be overestimated [3]. Some of the large molecular weight sugars have been proposed as external markers, but it will be necessary to show that they are not taken up by the metabolically active cells in the airways.

An internal marker is a substance in the lung lining fluid that is not present in the introduced fluid. If the concentration of the marker in the lung lining fluid and aspirated fluid is known, as well as the volume of aspirated fluid, it is possible to calculate the volume of aspirated lung lining fluid. By selecting substances that are freely permeable into extravascular spaces, the concentration of the internal marker is assumed to be the same in the blood and the lung lining fluid. However, care must to be taken to avoid a marker that may be secreted or produced in the lung lining fluid, such as immunoglobulin (Ig) A, and would, therefore, not be a valid marker. Urea has been commonly used as an internal marker [4].

The major problem with internal markers is that they may cross into the fluid during the procedure. Since the concentration of the marker is zero in the introduced fluid, the lavage process will dilute the marker in the lung lining fluid. During the aspiration process, the marker may be secreted into the fluid. It has been shown that the total amount of urea aspirated increases significantly as the time to perform the aspiration increases. This increased amount will increase the concentration in the aspirated fluid [5-7]. This will be interpreted as an 
increase in the amount of lung lining fluid found in the aspirated fluid.

Albumin is a large-molecular-weight protein that does not cross the membrane rapidly. It is found in the lung and other extracellular spaces at a reduced concentration compared to the blood. Increased albumin concentration has been a well accepted marker of inflammation, used, for example, to distinguish transudates from exudates in the pleural space. In measuring the concentration of albumin in the BAL fluid, it has been noted that albumin concentration is higher in patients with interstitial lung disease than in controls [2,8]. The highest concentrations are found in patients with ARDS [9]. Albumin concentration in BAL fluid has been proposed as a marker of increased permeability of the alveolar space in inflammation.

WARD et al. [10] have demonstrated that the lavage process may also affect the results. They examined the cause of increased albumin in the BAL fluid of patients with interstitial lung disease (ILD). By giving radioactive albumin shortly before the procedure, they could measure the amount of albumin that crosses into the lung during the lavage process. They postulated that the procedure itself, with the high filling volumes and high negative pressures during aspiration, could lead to increased permeability. Thus, a technique providing a better sampling of the alveolar space leads to a change in the concentration in the space.

Given the increased permeability seen in ILD, it would be expected that the increased albumin would be due in part to increased leakage during lavage. In the current issue of the Journal, WARD et al. [11] report on albumin in the BAL fluid of patients with ILD.They demonstrated rapid transport of albumin into the lung of some, but not all, healthy volunteers, similar to their previous findings [10]. This supports their view that the lavage process can lead to leakage of serum albumin into the lung. The investigators did find an increase in albumin in their patients with ILD. However only some patients had significant leakage of radioactive albumin, an indicator of leakage during the lavage procedure. The percentage of patients with leakage and the proportion of leakage was the same for the healthy controls and the patients with ILD. Thus, the increased albumin generally found in ILD must be due to increased concentration of albumin in the lung lining fluid. The actual concentration of albumin in the lung lining fluid remains unknown since the lavage process may change the concentration in the aspirated fluid.

Given all these problems, is there any role for BAL as a research or diagnostic procedure? The identification of tumour cells or certain microorganisms (e.g. Pneumocystis carinii, Mycobacterium tuberculosis) is always abnormal, and concentration is irrelevant. Other items can be measured in proportion to each other in the aspirated fluid. For example, an increased percentage of eosinophils is useful in diagnosing eosinophilic pneumonia. The proportion of lymphocyte subpopulations has been used to support the diagnosis of sarcoidosis. The concentration of a solute may also be relevant. Cytokines released during the inflammatory response of ARDS, for example, may go from undetectable levels to a 1,000 fold excess [9]. Differences of greater than a 100 fold between groups are unlikely to be the result of differences in the volume of aspirated fluid. Therefore, a 100 fold or greater difference in the concentration of a solute is probably due to the disease process, especially if the lavage procedure has been the same for both groups.

A European Respiratory Society (ERS)-supported task force has been trying to establish a method for reporting the acellular component of BAL. Some of the recommendations of that task-force were presented at the annual ERS meeting in Stockholm in 1996, including reporting per millilitre of BAL fluid. A pragmatic approach to reporting concentrations in BAL fluid has been proposed by this group. It is hoped that this recommendation will be adopted to enhance communication between laboratories.

One of the rationales for choosing this reporting technique is the utility of BAL to diagnose bacterial pneumonia. The original method reported by ourselves and others used the unconcentrated BAL fluid and expressed the amount of bacteria as colony forming units $\cdot \mathrm{mL}^{-1}$ of BAL fluid [12]. Studies from groups in Europe and the USA have shown clear-cut differences in the concentration of bacteria in BAL fluid between patients who had pneumonia and those who did not $[13,14]$. These conclusions have been verified by independent measures of pneumonia, such as pathological specimens $[14,15]$.

As one examines the lavage process closely, one can appreciate the uncertainty a physician may have about the retrieval process. As WARD et al. [11] pointed out, the lavage procedure itself affects the results. Also, the lung is an active organ, secreting and absorbing proteins and cells during the lavage procedure. I doubt that we will ever resolve the uncertainties of dilution that occur during lavage. However, as the American pragmatist Ben Franklin once observed, "The only thing certain in life is death and taxes."

\section{References}

1. Reynolds HY, Newball HH. Analysis of proteins and respiratory cells obtained from human lungs by bronchial lavage. J Lab Clin Med 1974; 84: 559-573.

2. Baughman RP, Bosken CH, Loudon RG, Hurtubise P, Wesseler T. Quantitation of bronchoalveolar lavage with methylene blue. Am Rev Respir Dis 1983; 128: 266-270.

3. Von Wichert P, Joseph K, Muller B, Franck WM. Bronchoalveolar lavage. Quantitation of intra-alveolar fluid? Am Rev Respir Dis 1993; 147: 148-152.

4. Rennard SI, Basset G, Lecossier D, O'Donnell K, Martin $\mathrm{P}$, Crystal RG. Estimation of volume of epithelial lining fluid recovered by lavage using urea as marker of dilution. J Appl Physiol 1986; 60: 532-538.

5. Marcy TW, Merrill W, Rankin JA, Reynolds HY. Limitations of using urea to quantify epithelial lining fluid recovered by bronchoalveolar lavage. Am Rev Respir Dis 1987; 135: 1276-1280.

6. Schmekel B, Hornblad Y, Hvatum M, Norlund AL, Venge P. Kinetic retrieval of eosinophil cationic protein, hyaluronan, secretory IgA, albumin, and urea during BAL in healthy subjects. Chest 1995; 108: 62-67.

7. van de Graaf EA, Jansen HM, Weber JA, Koolen MG, Out TA. Influx of urea during bronchoalveolar lavage 
depends on the permeability of the respiratory membrane. Clin Chim Acta 1991; 196: 27-39.

8. Jones KP, Edwards JH, Reynolds SP, Peters TJ, Davies $\mathrm{BH}$. A comparison of albumin and urea as reference markers in bronchoalveolar lavage fluid from patients with interstitial lung disease. Eur Respir J 1990; 3: 152156.

9. Baughman RP, Gunther KL, Rashkin MC, Keeton DA, Pattishall EN. Changes in the inflammatory response of the lung during acute respiratory distress syndrome: prognostic indicators. Am J Respir Crit Care Med 1996; 154: 76-81.

10. Ward C, Duddridge M, Fenwick J, et al. The origin of water and urea sampled at bronchoalveolar lavage in asthmatic and control subjects. Am Rev Respir Dis 1992; 146: 444-447.

11. Ward C, Fenwick J, Booth H, Walters EH. Albumin is not suitable as a marker of bronchoalveolar lavage (BAL) dilution in interstitial lung disease (ILD). Eur Respir $J$ 1997; 2029-2033.

12. Thorpe JE, Baughman RP, Frame PT, Wesseler TA, Staneck JL. Bronchoalveolar lavage for diagnosing acute bacterial pneumonia. J Infect Dis 1987; 155: 855-861.

13. Cantral DE, Tape TG, Reed EC, Spurzem JR, Rennard SI, Thompson AB. Quantitative culture of bronchoalveolar lavage fluid for the diagnosis of bacterial pneumonia. Am J Med 1993; 95: 601-607.

14. Torres A, el Ebiary M, Padro L, et al. Validation of different techniques for the diagnosis of ventilator-associated pneumonia. Comparison with immediate postmortem pulmonary biopsy. Am J Respir Crit Care Med 1994; 149: 324-331.

15. Chastre J, Fagon JY, Bornet Lecso M, et al. Evaluation of bronchoscopic techniques for the diagnosis of nosocomial pneumonia. Am J Respir Crit Care Med 1995; 152: 231-240. 